\title{
Détection des radionucléides artificiels dans les produits laitiers après l'accident de Tchernobyl*
}

\author{
F. JANIN, G. LEPROVOST, C. LAPEYRE, A. GUIARD**
}

(Manuscrit reçu le 25 novembre 1987)

\begin{abstract}
RÉSUMÉ
Dès les premiers jours du mois de mai 1986, le contrôle du niveau de radioactivité artificielle dans les denrées alimentaires a été intensifié et de nombreuses mesures d'activité ont été réalisées dans les laboratoires des services vétérinaires.

Une évaluation des activités en iode a été faite à partir de 6 prélèvements de lait fournis par chaque département. Les activités moyennes en iode 131 sont restées inférieures à $30 \mathrm{~Bq} / \mathrm{kg}$ dans toute la moitié Ouest de la France. Des niveaux plus élevés ont été détectés dans l'Est. Les valeurs maximales enregistrées ont été de $700 \mathrm{~Bq} / \mathrm{kg}$ dans des échantillons du Nord-Est de la France, au cours de la première semaine du mois de mai 1986 . Les valeurs les plus élevées en césium 137 et 134 ont été mises en évidence dans le Sud-Est oủ des pluies diluviennes sont survenues au début du mois de mai. Ces précipitations ont entraîné des dépôts d'isotopes radioactifs plus importants dans ces régions. Cependant, une très grande hétérogénéité des activités a été observée dans des zones très proches. Les valeurs moyennes ont toujours été très inférieures aux niveaux de tolérance maximale fixées par la CEE. Aucun produit animal ou d'origine animale n'a été retiré à la consommation.
\end{abstract}

\section{ABSTRACT}

Since the first days of May 1986, the control of radioactivity levels in food has been intensified. Activity levels were determined in two series of milk sampling from every department. lodine 131 mean levels in western France were below $30 \mathrm{~Bq} / \mathrm{kg}$ but above this value in the eastern part. The highest value was $700 \mathrm{~Bq} / \mathrm{kg}$, recorded in Northeastern France during the first week of May. The highest levels in cesium 137 and cesium 134 were found in Southeastern France where heavy rain storms were recorded in early May.

Radioactivity levels of iodine 131, cesium 137 and cesium 134 were recorded all the year long: an increase of cesium amounts was noticed during Winter when the cattle was fed with grass cropped in May and June 1986.

The recorded radioactivity levels were always below the EEC regulatory action levels, therefore no dairy products were withdrawn from the market.

* Communication présentée lors du congrès franco-italien SFRP-Associazione italiana di protezione contro le radiazioni, Castelgandolfo (Italie), 12-13 octobre 1987.

** Laboratoire central d'hygiène alimentaire, Direction générale de l'alimentation, ministère de l'Agriculture, 43, rue de Dantzig, 75015 Paris. 


\section{INTRODUCTION}

La Direction générale de l'alimentation du ministère de l'Agriculture contrôle les niveaux de radionucléides présents dans les denrées alimentaires animales et d'origine animale; 17 laboratoires des services vétérinaires départementaux participent au programme de surveillance depuis 1970.

Dès que l'accident de Tchernobyl qui s'est produit dans la nuit du 26 au 27 avril 1986, a été connu, le contrôle du lait a été intensifié afin de vérifier que les activités détectées dans cette denrée restaient acceptables quel que soit le lieu de production.

Les principaux radionucléides déposés sur le sol en Europe occidentale, et qui sont toxiques par ingestion, étaient l'iode 131, le césium 137 et le césium 134 [1]. Les radionucléides peu volatiles tels que le strontium ou des émetteurs alpha comme le plutonium ont représenté une toute petite fraction de la composition du nuage radioactif.

L'iode 131, dont la période est de 8 jours, a été détecté dans l'environnement pendant un mois. Ce radionucléide se concentre dans la thyroïde en provoquant une irradiation directe des cellules de cet organe [7]. La quantité d'iode fixé est limitée. L'excédent, qui est ingéré, est éliminé par voie urinaire et par le lait. Le transfert de l'iode vers l'enfant en bas âge [2] se fait principalement par l'ingestion de lait. Les jeunes enfants représentent une population critique en raison de leur poids, de leur alimentation lactée et du rôle de la thyroïde pendant la croissance. Dans l'interprétation dosimétrique, leur cas est abordé avec des facteurs de dose appropriés qui tiennent compte des dimensions de la thyroïde et du métabolisme différent de celui des adultes.

Le césium se répartit dans les muscles et dans les tissus mous, au niveau du liquide cellulaire. On le retrouve dans tout l'organisme. Le métabolisme du césium est le même que celui du potassium. Ce radionucléide passe également dans le lait. La période de l'isotope 137 est de 30 ans, sa période biologique a été estimée entre 70 et 150 jours. Le césium 134 a une période de 2 ans. A noter qu'avant l'accident de Tchernobyl, des traces de césium 137 provenant des essais nucléaires antérieurs subsistaient déjà, à de très bas niveaux dans certains produits agricoles de l'hémisphère nord.

\section{RÉGLEMENTATION MISE EN PLACE APRĖS L'ACCIDENT POUR MAINTENIR LES ÉCHANGES DE DENRÉES ALIMENTAIRES ET GARANTIR LA QUALITÉ HYGIÉNIQUE DES PRODUITS FRANÇAIS}

Dès que l'accident a èté notifié, une commission post-accidentelle a été mise en place à la Direction générale de l'alimentation. II a été décidé d'intensifier les mesures d'activité dans les produits animaux et d'origine animale et de suspendre les importations de produits en provenance de régions situées à moins de $1000 \mathrm{~km}$ du lieu de l'accident. 
DÉTECTION DES RADIONUCLEIDES ARTIFICIELS DANS LES PRODUITS LAITIERS APRES TCHERNOBYL

Au niveau communautaire, des recommandations parues le 6 mai 1986 ont établi les activités maximales en iode 131 par kilogramme de produit (tableau I). Les importations de denrées en provenance de 9 pays ont été suspendues.

TABLEAU I

Niveaux de tolérance maximale en iode 131 en mai 1986

\begin{tabular}{|r|c|c|}
\hline \multirow{2}{*}{6} & $\begin{array}{c}\text { Lait et produits laitiers } \\
\text { en } \mathrm{Bq} / \mathrm{kg}\end{array}$ & $\begin{array}{c}\text { Fruits et légumes } \\
\text { en } \mathrm{Bq} / \mathrm{kg}\end{array}$ \\
\cline { 2 - 3 } 16 mai & 500 & 350 \\
$22 \mathrm{mai}$ & 250 & 175 \\
& 125 & 90 \\
\hline
\end{tabular}

La France s'est engagée à appliquer ces recommandations qui n'ont pas fait l'unanimité entre les États membres. Elles ont été jugées trop élevées par certains (RFA, Luxembourg, Pays Bas...).La France et la Grande-Bretagne, en revanche, les ont jugées sans fondement toxicologique sur le plan de l'interprétation sanitaire et trop éloignées des recommandations de la CIPR [6] et même des recommandations communautaires pour limiter les doses d'irradiations en cas de dissémination de produits radioactifs [3]. Cependant, la définition de niveaux de tolérance maximale admissible par kilogramme de produit a été acceptée par la France et une surveillance par sondage des produits nationaux et des produits importés a été instaurée à partir du 6 mai 1986.

Le 17 juin, un nouveau règlement a été notifié par la Commission des Communautés européennes, qui levait l'interdiction d'importer des produits en provenance de pays situés à moins de $1000 \mathrm{~km}$ du lieu de l'accident. Le 17 juin un avis aux importateurs a défini des niveaux de tolérance maximale pour les activités en césium 137 et en césium 134. La réouverture des frontières a été décidée après examen des résultats de mesures fournis par les différents pays et en exigeant, pour chaque importation, un certificat établi après contrôle du respect des exigences communautaires (tableau II).

\section{TABLEAU ॥}

\section{Niveaux de tolérance maximale pour les activités ajoutées en césium 134 et en césium 137 établis par la CEE [5]}

- $370 \mathrm{~Bq}$ par $\mathrm{kg}$ pour le lait et les produits laitiers destinés aux nourrissons de moins de six mois;

$-600 \mathrm{~Bq}$ par $\mathrm{kg}$ pour tous les autres produits.

Malgré les dispositions communautaires déjà très prudentes pour éviter une exposition supplémentaire à la suite de l'ingestion de denrées contaminées, des pays tiers ont imposé l'absence complète de radionucléides artificiels dans les produits alimentaires. 
C'est pour répondre aux demandes d'informations sur le niveau de contamination des produits français et pour maintenir les échanges commerciaux que de nombreuses mesures ont été faites par les laboratoires des services vétérinaires.

\section{MATÉRIEL ET MÉTHODES}

Immédiatement après l'accident, la composition en radionucléides des retombés parvenues jusqu'en Europe occidentale a montré que ceux qui pouvaient présenter un danger pour le consommateur étaient tous des radionucléides émetteurs de rayonnements bêta et gamma.

Ce sont les isotopes de l'iode et du césium qui pouvaient éventuellement nécessiter des restrictions de consommation.

\section{APPAREILS DE MESURE}

Des ensembles de spectrométrie gamma ont été utilisés dans tous les laboratoires avec des détecteurs à l'iodure de sodium ou des semi-conducteurs en germanium. Les activités en iode 131, en césium 137 et césium 134 ont été exprimées en $\mathrm{Bq} / \mathrm{kg}$ de produit.

Les mesures ont été pratiquées directement sur les laits liquides et les poudres de lait, chaque échantillon étant placé dans un récipient de géométrie constante. L'étalonnage a été réalisé dans les mêmes conditions. Les fromages ont été broyés puis disposés dans des.récipients cylindriques.

\section{ÉCHANTILLONNAGE}

Les premiers jours du mois de mai 1986, quatre prélèvements de lait cru ont été demandés à chaque département. Deux autres prélèvements par département ont été analysés la deuxième quinzaine de mai pour évaluer les activités moyennes dues à l'iode, au césium 137 et au césium 134 sur tout le territoire.

Les activités dans les poudres de lait ont été suivies journellement afin de contrôler les productions des principales usines françaises pendant les mois de mai et de juin. Une surveillance régulière est toujours assurée conformément aux directives communautaires.

Des mesures d'activité sur les produits frais, les fromages et les beurres ont permis de suivre les répercussions de l'accident sur l'ensemble des productions laitières.

\section{RÉSULTATS}

Les premières mesures par spectrométrie gamma ont révélé la présence dans l'air et sur le sol de plusieurs isotopes de produits de fission ou d'activation. Les premiers jours du mois de mai, l'activité due aux isotopes de l'iode et du tellure représentaient $9 / 10$ de l'activité due à des isotopes artificiels (1). L'iode et le césium sont passés très rapidement dans les laits.

\section{ÉVALUATION des NIVEauX D'ACTIVITÉ des LaITS CRUS PAR L'IODE 131 ET LES ISOTOPES DU CÉSIUM}

Afin de pouvoir estimer rapidement le degré de contamination des laits nous avons réalisé des spectres gamma sur des laits provenant de productions d'animaux à l'herbage. 
La figure 1 montre un spectre gamma obtenu à partir d'un lait cru caractéristique de l'activité des laits français après l'accident de Tchernobyl. Dès le début des contrôles, une grande différence a été observée entre les laits des régions est et ouest. Dans toute la moitié ouest (Bretagne, Normandie, Pays-de-Loire et Sud-Est) les activités sont restées proches de la limite de détection. En revanche, dans la moitié est, l'iode 131 a été décelé dans les laits produits par les animaux à l'herbage au moment de l'accident, et les activités en iode 131 ont dépassé $100 \mathrm{~Bq} /$ kg. Sur la figure 2, sont rapportées les activités en iode 131. Cette carte a été complétée avec les moyennes calculées à partir de 6 prélèvements par département ( 2 laits de mélange, 4 laits individuels). Les activités en iode ont été ramenées au jour de prélèvement. Le seuil de détection pour l'iode 131 a èté fixé à $10 \mathrm{~Bq} / \mathrm{kg}$ pour tous les laboratoires. Les activités maximales en iode 131 étaient de $700 \mathrm{~Bq} / \mathrm{kg}$.

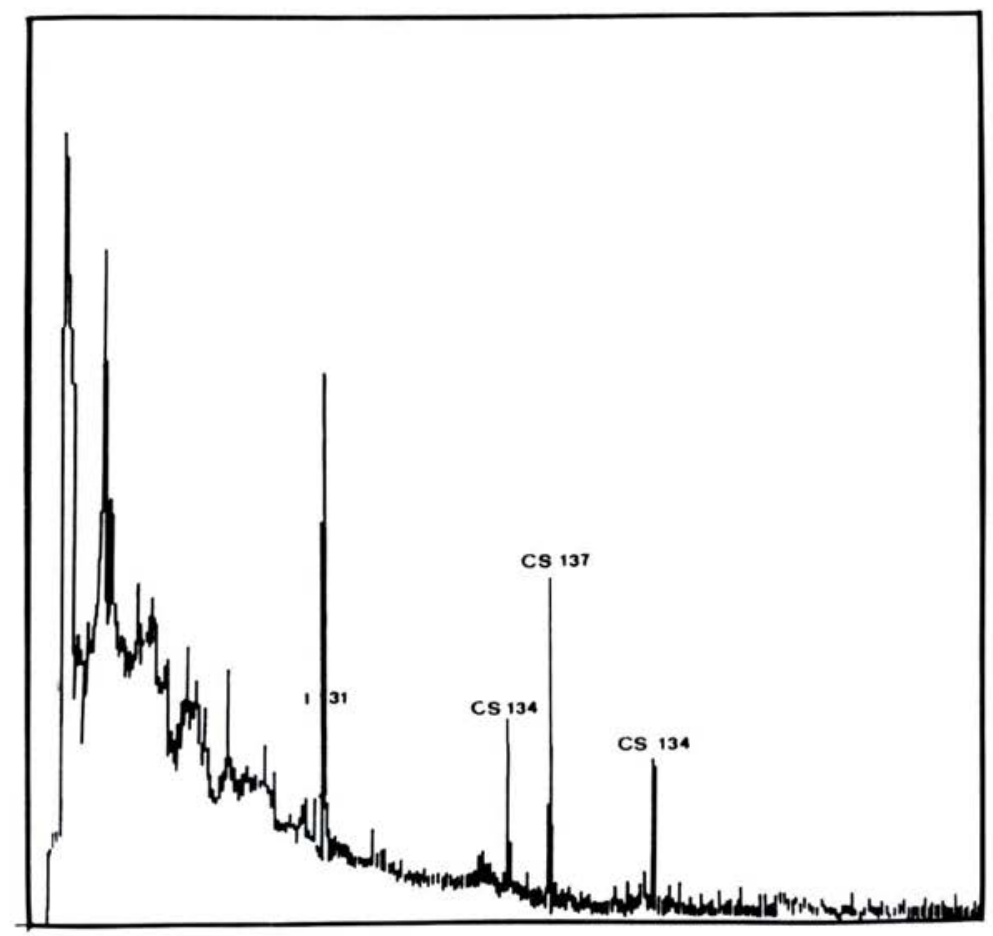

Fig. 1. - Spectre gamma, réalisé avec un détecteur germanium, d'un lait cru prélevé début mai dans le Sud-Est de la France. 


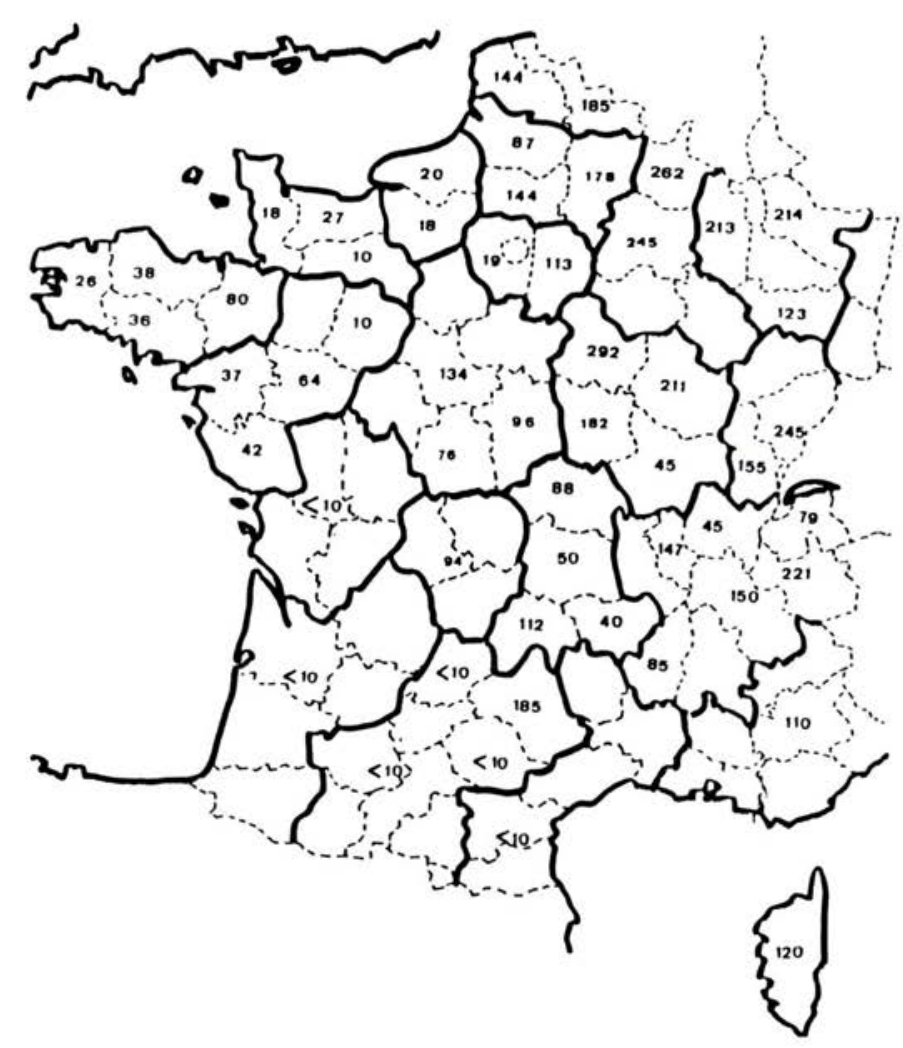

Fig. 2. - Moyenne des activités en iode 131, mesurées dans les laits français, début mai, calculée à partir de 6 échantillons par département.

Dans les échantillons où les activités en iode 131 ont dépassé $200 \mathrm{~Bq} / \mathrm{kg}$, des traces de césium 137 et 134 ont été mises en évidence relativement tôt. Dès la deuxième quinzaine du mois de mai, les activités en iode ont diminué pour atteindre des valeurs moyennes inférieures à $100 \mathrm{~Bq} / \mathrm{kg}$.

Les activités maximales (fig. 3) pour l'iode, le césium 137 et 134 dans les laits frais, montrent qu'à partir du mois de juin c'est le césium qui est encore présent en tant que radionucléide artificiel, l'activité due au césium 134 représente environ $50 \%$ de l'activité due au césium 137. La diminution des activités de ces deux isotopes a été plus lente, mais les activités détectées sont toujours restées très inférieures aux tolérances maximales communautaires. On a observé une remontée des activités en césium au début de l'hiver 1986-1987 lorsque les animaux sont rentrés à l'étable et ont été alimentés avec des ensilages et des fourrages collectés en mai et juin 1986. 


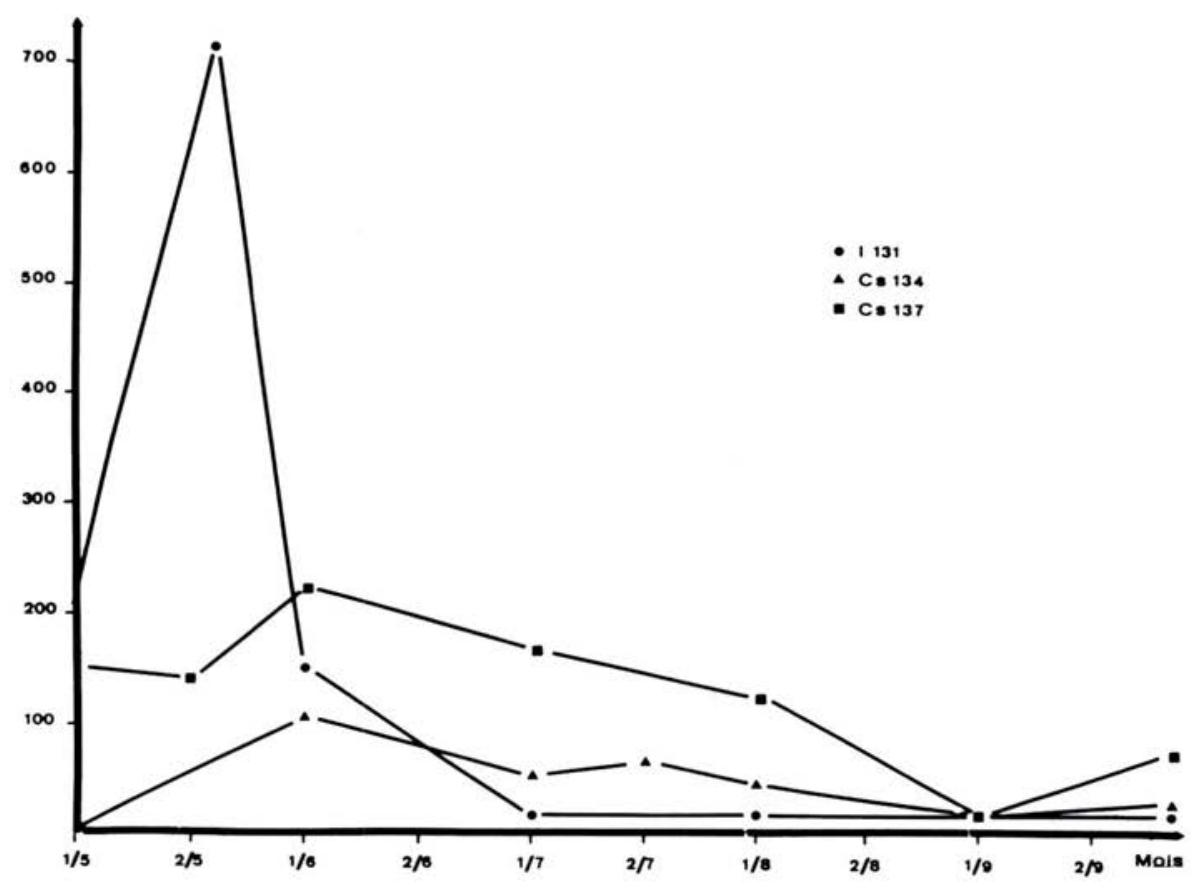

Fig. 3. - Valeurs maximales en iode 131, césium 137 et césium 134 détectées dans les laits français de mai à octobre 1986.

Les activités en césium 137 et 134 sont restées inférieures au seuil de mesure dans les régions Ouest. Le seuil de détection pour les activités en césium 137 et 134 a été fixé à $30 \mathrm{~Bq} / \mathrm{kg}$.

Les activités en césium ont été nettement plus élevées dans les zones où d'importantes précipitations se sont produites début mai. La somme des activités en césium 134 et en césium 137 a été très hétérogène, en des points de prélèvements proches. L'enquête réalisée dans le département de la Drôme en est un exemple, puisque les activités mesurées à partir de foins ont varié de 100 à plus $10000 \mathrm{~Bq} / \mathrm{kg}$ à des distances inférieures à $100 \mathrm{~km}$. On a retrouvé des écarts similaires dans les laits.

\section{MESURES DANS LES LAITS DE CHĖVRE ET DE BREBIS}

Dans les laits de chèvre et de brebis une contamination notablement plus élevée que dans le lait de vache a été constatée dans les régions du sud-est de la France. Certains laits ont eu des activités en iode 131 qui ont dépassé $1000 \mathrm{~Bq} / \mathrm{kg}$ pendant la première semaine du mois de mai. Cependant, comme pour le lait de vache, une grande hétérogénéité a été observée. 
Les activités les plus élevées ont été mesurées dans les laits prélevés dans des élevages traditionnels où les animaux sont en liberté. Le mode d'alimentation de ces animaux, qui broutent des herbes rases et qui ont donc collecté des végétaux sur de grandes surfaces explique les activités relativement plus élevées que celles mesurées dans le lait de vache. Une surveillance a été exercée sur les productions de la Drôme, l'Ardèche, l'Aveyron et la Corse. Sur la figure 4, les activités moyennes détectées dans ces produits sont rapportées en fonction du temps. La contamination par le césium a été suivie pendant plusieurs mois. Les activités relativement plus importantes ont parfois dépassées, les tolérances communautaires, ces produits n'ont néanmoins pas donné lieu a des restrictions de consommation. Les laitages de chèvre et de brebis ne sont pas destinés aux très jeunes enfants. Les activités en iode 131 qui ont dépassé $500 \mathrm{~Bq}$ par $\mathrm{kg}$ pendant quelques jours, n'ont été mesurées que dans un très petit nombre d'échantillons.

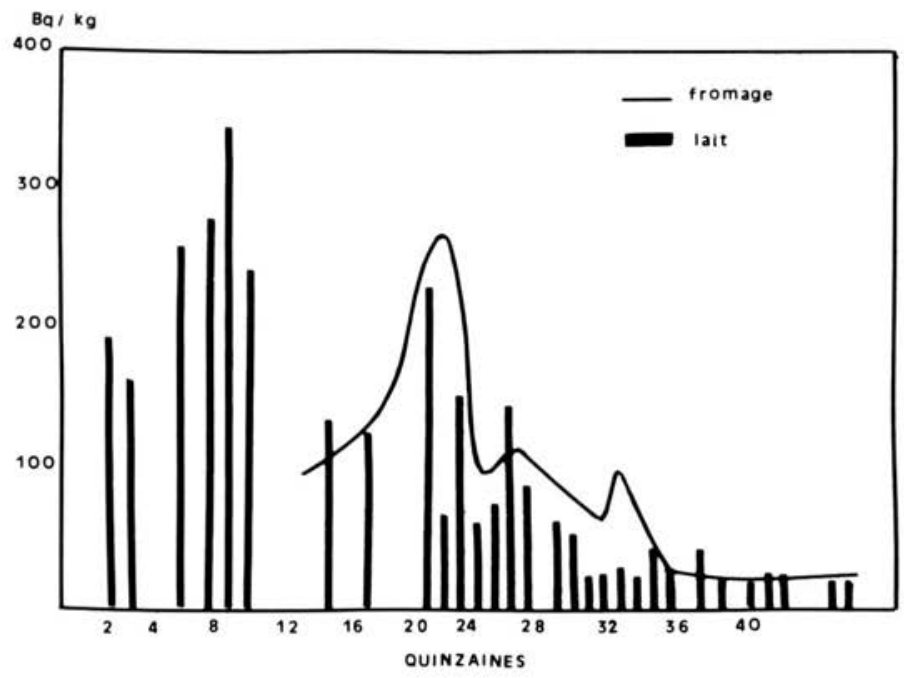

Fig. 4. - Activités en césium 137 et 134 mesurées dans les laits de chèvre et de brebis pendant les mois qui ont suivi l'accident de Tchernobyl.

\section{ÉTUDE DES LAITS EN POUDRE}

La production de plusieurs usines de poudre de lait a été contrôlée dès que l'accident a été connu, et en particulier le lait destiné aux nourrissons. Celui-ci est toujours soumis à une surveillance régulière, conformément à la réglementation en vigueur, pour la commercialisation sur le territoire français et pour l'exportation. 
Les poudres de lait collectées dans les régions de l'ouest de la France ont été très peu contaminées et les activités moyennes en iode et en césium sont restées inférieures à $30 \mathrm{~Bq} / \mathrm{kg}$ de poudre. Dans le nord, des activités plus élevées ont été mesurées mais rapportées au litre de lait reconstitué, elles sont restées inférieures aux tolérances communautaires (fig. 5).

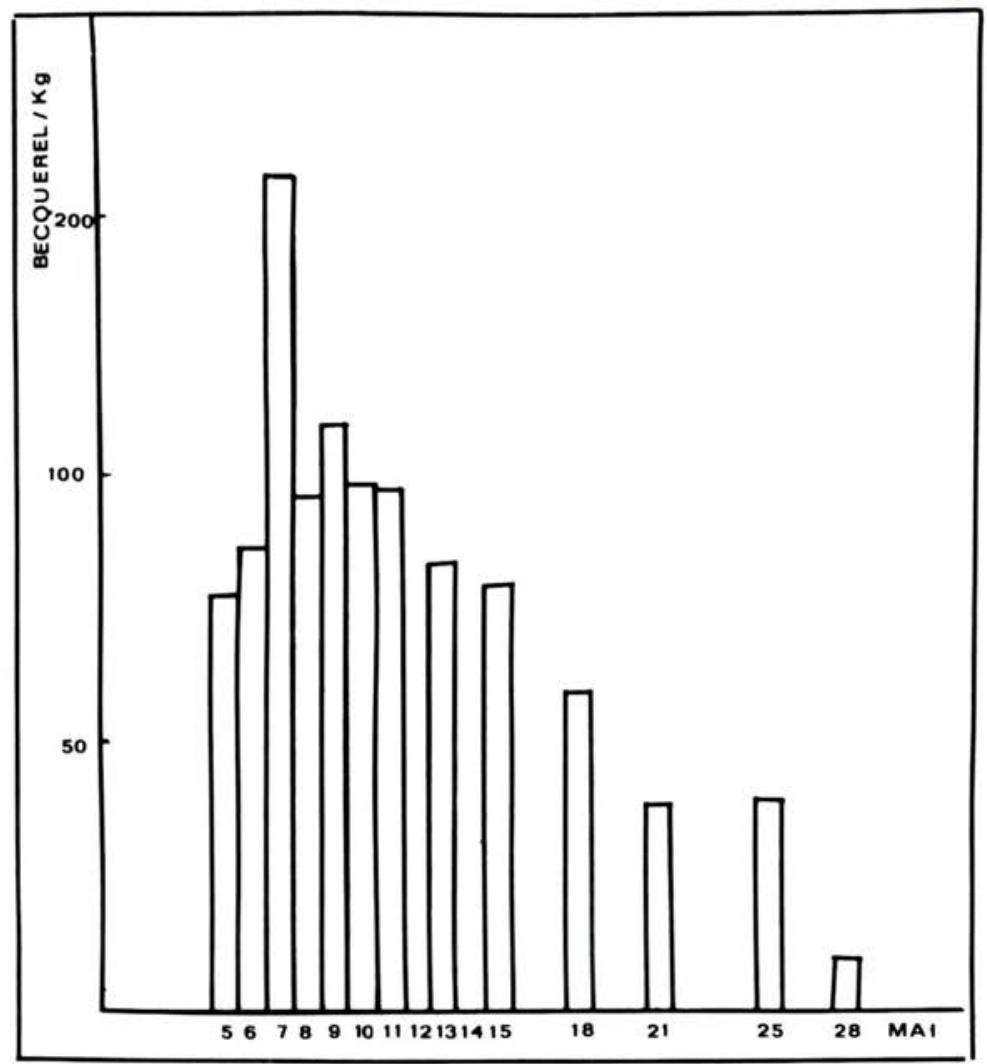

Fig. 5. - Etudes de la contamination de poudres de lait en provenance d'une même usine de la région nord. L'activité est évaluée par kilogramme de poudre (environ 8 I sont nécessaires pour produire $1 \mathrm{~kg}$ de poudre).

Sur ce schéma sont rapportées les sommes des activités en césium 134 et en césium 137 avec, en abscisse, les jours de fabrication.

Sur la figure 6, sont rapportées les activités en iode 131, césium 134 et césium 137 mesurées dans des poudres de lait de l'est de la France du début mai 1986 à août 1987. Au début de l'hiver 1986, les activités en césium sont remontées sans jamais atteindre les niveaux de mai-juin. 


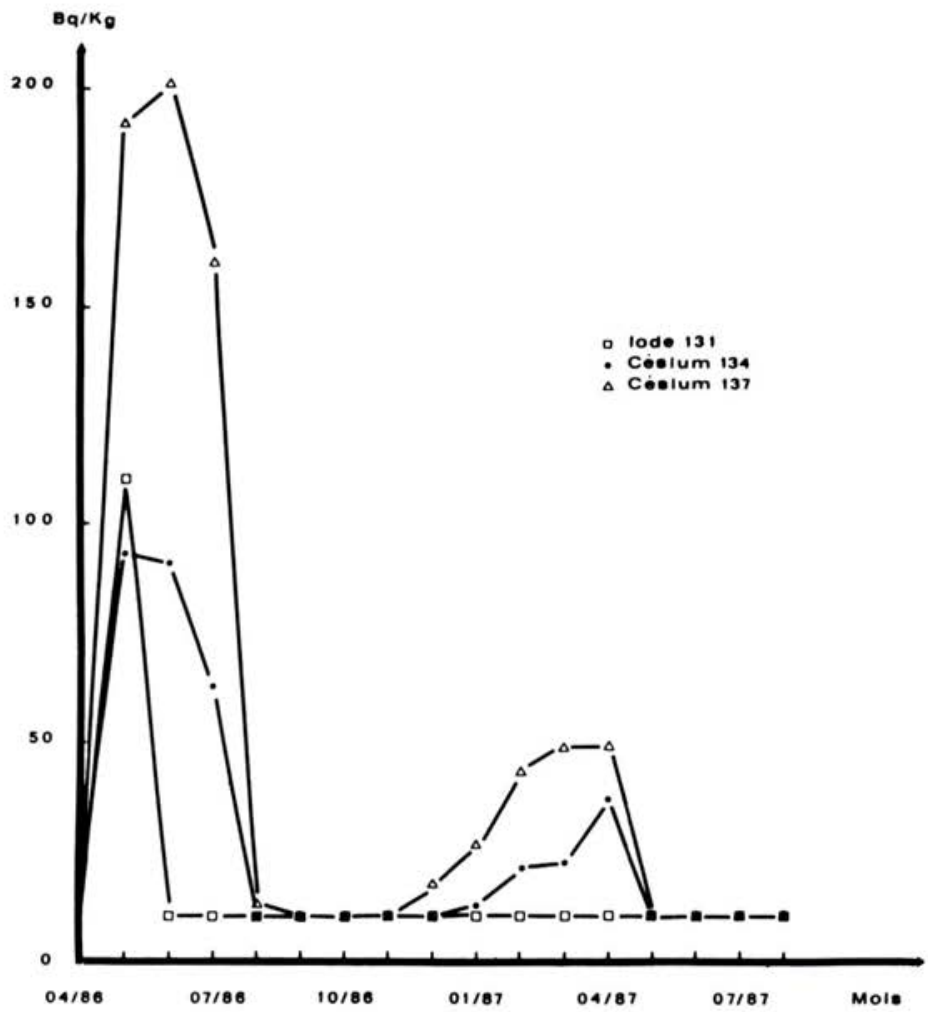

Fig. 6. - Evolution des activités en iode 131, césium 134 et césium 137 dans des poudres de lait préparées à partir de laits collectés dans l'est de la France. L'activité par radionucléide est exprimée en becquerel par kilogramme de poudre (1 kg de poudre est préparé avec environ 8 I de lait).

\section{PRODUITS LAITIERS}

\section{Produits frais}

Les valeurs moyennes n'ont pas dépassé $50 \mathrm{~Bq} / \mathrm{kg}$. Début mai, en effet, une grande proportion de vaches laitières étaient encore à l'étable et les laits de grand mélange ont été peu contaminés. Les activités des produits frais industriels contrôlés sont toutes restées inférieures à $50 \mathrm{~Bq} / \mathrm{kg}$.

\section{Les fromages}

De nombreuses espèces ont été étudiées. Dans la majorité des échantillons, les activités en iode et en césium sont restées inférieures au seuil de détection. Les activités les plus élevées ont été observées dans du fromage de chèvre et de brebis. La plupart des fromages sont consommés plusieurs 
jours ou même plusieurs semaines après la date de fabrication, et l'iode présent dans les laits les premiers jours après l'accident, compte tenu de sa période, a rapidement décru. L'activité en iode n'a pas atteint la limite communautaire dans les produits français. Le césium, qui est soluble, est entraîné pour une part plus ou moins importante, suivant la technologie de préparation dans le lactoserum. Sur la figure 4, à côté des activités des laits, sont indiquées des activités mesurées dans des fromages de chèvre et de brebis. Le césium n'est pas réellement concentré dans les fromages, un fromage de la taille d'un camembert nécessite 3 I de lait, les activités mesurées dans ce fromage de grande consommation, sont restées inférieures à $50 \mathrm{~Bq} / \mathrm{kg}$ pour les deux césium.

\section{DISCUSSION}

Les résultats présentés dans cette étude ont été rassemblés à la suite d'analyses réalisées dans les différents laboratoires des services vétérinaires du ministère de l'Agriculture, à la demande de la Direction générale de l'alimentation, pour évaluer les niveaux de contamination dans l'ensemble des produits alimentaires français, le lait étant, de loin, le vecteur principal de l'iode et des césium vers l'homme.

Dans les jours qui ont suivi la dissémination de radionucléides, de nombreux industriels du secteur agro-alimentaire nous ont sollicités pour que des mesures d'activité soient réalisées dans les matières premières utilisées pour la préparation de leurs produits à base de lait.

A partir du mois d'octobre 1986, ce sont surtout les exigences des pays importateurs de produits agricoles français qui ont été à l'origine des contrôles.

Parmi les méthodes de détection proposées pour rechercher les radionucléides artificiels, la spectrométrie gamma a permis de traiter un nombre élevé d'échantillons et de répondre aux nombreuses demandes d'examens.

L'évaluation des activités en iode et en césium, dans toutes sortes de produits, a permis de transmettre à la Commission des Communautés européennes des valeurs moyennes et des valeurs maximales, par catégorie de produits, d'abord quotidiennes, puis hebdomadaires et enfin mensuelles. Ces données ajoutées à celles produites par les Services de la répression des fraudes pour les végétaux et par le Service central de protection contre les rayonnements ionisants, ont permis de maintenir normalement les exportations vers les Etats membres du Marché commun.

\section{CONCLUSIONS}

La répercussion la plus importante de l'accident de Tchernobyl en France a été la contamination des denrées alimentaires par l'iode 131, le césium 137 et le césium 134. 19000 analyses ont été faites par les VOL. $23-\mathrm{N}^{\circ} 2$ 
laboratoires des services vétérinaires dont 10000 sur des produits laitiers. Les différentes études ont montré que:

- les laits crus collectés pendant la période qui a suivi l'accident pouvaient être consommés sans aucune restriction;

- l'iode 131 a entraîné une exposition supplémentaire significative pendant quelques jours, la période effective de l'iode 131, dans le lait, a été évaluée à 5 jours;

- le passage des deux isotopes du césium dans le lait a été décalé par rapport à celui de l'iode et les valeurs maximales ont été détectées dans le Nord-Est au début de l'été 1986; une remontée des activités des deux isotopes du césium a été observée au début de l'hiver 86-87;

- plus de 5000 analyses ont été faites avec des limites de détection basses, inférieures à $10 \mathrm{~Bq} / \mathrm{kg}$, dans des produits alimentaires français afin de permettre leur exportation vers des pays tiers qui exigent des activités en césium 137 et en césium 134 inférieures à 25 et même à $10 \mathrm{~Bq} / \mathrm{kg}$.

Cet accident a mis en évidence la nécessité de disposer de nombreux laboratoires, dispersés sur l'ensemble du territoire, pour détecter immédiatement l'iode et les isotopes du césium pendant une période beaucoup plus longue. La durée de l'analyse doit être courte pour ne pas ralentir la commercialisation de denrées périssables. Les exigences quant au seuil de sensibilité limitent l'emploi de détecteurs portables qui permettent les autocontrôles.

D'autres laboratoires vétérinaires ont reçu un équipement en spectrométrie gamma, 17 sont opérationnels fin 1987 et participent au plan de surveillance des denrées alimentaires animales et d'origine animale. TESOLIN).

(Collaboration technique : Muriel BOREL, Cyril HENRY et Béatrice

\section{RÉFÉRENCES}

[1] COMMISSARIAT À L'ÉNERGIE ATOMIQUE. INSTITUT DE PROTECTION ET DE SÛRETÉ NUCLÉAIRE (IPSN). L'accident de Tchernobyl. Rapport IPSN 2/86, rev. 3 , 1986.

[2] COMMISSION DES COMMUNAUTÉS EUROPÉENNES. Méthodologie pour l'évaluation des conséquences radiologiques des rejets d'effluents radioactifs en fonctionnement normal. Rapport établi conjointement par le Commissariat à l'énergie atomique et le National radiological protection board. Rapport V/3865/79. Luxembourg: CCE, 1979.

[3] COMMISSION DES COMMUNAUTÉS EUROPÉENNES. Radiological protection criteria for controlling doses in the event of accidental releases of radioactive material. Rapport V/5290/82 EN. Luxembourg: CCE, 1982. 
DÉTECTION DES RADIONUCLÉIDES ARTIFICIELS DANS LES PRODUITS LAITIERS APRÉS TCHERNOBYL

[4] COMMISSION DES COMMUNAUTÉS EUROPÉENNES. Recommandation du 6 mai 1986. Coordination des mesures nationales prises à l'égard des produits agricoles suite aux retombées radioactives provenant d'Union soviétique. General guidance on dose values for the introduction of countermeasures. Règlement CCE N. $86 / 156$.

[5] COMMISSION DES COMMUNAUTÉS EUROPÉENNES. Règlement (CEE) N 1762/ 86 du 5 juin 1986 portant modalités d'application du règlement (CEE) N. 1707/86 du Conseil relatif aux conditions d'importation de produits agricoles originaires des pays tiers à la suite de l'accident survenu à la centrale de Tchernobyl. JOCE 1986, L152, 41-44.

[6] INTERNATIONAL COMMISSION ON RADIOLOGICAL PROTECTION (ICRP). Recommendations... (ICRP Publication 26). Ann. ICRP, 1977, 1 (3). Oxford: Pergamon Press, 1977).

[7] TUBIANA M. Métabolisme et radiotoxicité de l'iode radioactif. In: Toxiques nucléaires (Galle P, Ed.). Paris: Masson, 1982. 